This is an electronic reprint of the original article. This reprint may differ from the original in pagination and typographic detail.

Author(s): Vockenhuber, Christof; Jensen, Jens; Julin, Jaakko; Kettunen, Heikki; Laitinen, Mikko; Rossi, Mikko; Sajavaara, Timo; Osmani, Orkhan; Schinner, Andreas; Sigmund, Peter; Whitlow, Harry

Title: $\quad$ Energy-loss straggling of 2-10 MeV/u Kr ions in gases

Year: $\quad 2013$

Version:

Please cite the original version:

Vockenhuber, C., Jensen, J., Julin, J., Kettunen, H., Laitinen, M., Rossi, M., Sajavaara, T., Osmani, O., Schinner, A., Sigmund, P., \& Whitlow, H. (2013). Energy-loss straggling of 2-10 MeV/u Kr ions in gases. European Physical Journal D, 67(7), Article 145. https://doi.org/10.1140/epjd/e2013-40095-4

All material supplied via JYX is protected by copyright and other intellectual property rights, and duplication or sale of all or part of any of the repository collections is not permitted, except that material may be duplicated by you for your research use or educational purposes in electronic or print form. You must obtain permission for any other use. Electronic or print copies may not be offered, whether for sale or otherwise to anyone who is not an authorised user. 


\title{
Energy-Loss Straggling of 2-10 MeV/u Kr ions in Gases
}

\author{
C. Vockenhuber ${ }^{2}$, J. Jensen ${ }^{3}$, J. Julin ${ }^{1}$, H. Kettunen ${ }^{1}$, M. Laitinen ${ }^{1}$, M. Rossi ${ }^{1}$, T. Sajavaara ${ }^{1}$, O. Osmani $^{4,7}$, A. $^{2}$ \\ Schinner $^{5}$, P. Sigmund ${ }^{6}$, and H. J. Whitlow ${ }^{1,8}$ \\ 1 Department of Physics, University of Jyväskylä, 40014 Jyväskylä, Finland \\ 2 Laboratory for Ion Beam Physics, ETH, 8093 Zürich, Switzerland \\ 3 Department of Physics, Chemistry and Biology, Linköping University, 58183 Linköping, Sweden \\ 4 Donostia International Physics Center, 20018 San Sebastian, Spain \\ 5 Department of Experimental Physics, Johannes Kepler University, 4040 Linz-Auhof, Austria \\ 6 Department of Physics, Chemistry and Pharmacy, University of Southern Denmark, 5230 Odense, Denmark \\ 7 Department of Physics, University of Duisburg-Essen, 47048 Duisburg, Germany (present address) \\ ${ }^{8}$ Institut des Microtechnologies Appliquées Arc, Haute Ecole Arc Ingénierie, 2300 La Chaux-de-Fonds, Switzerland (present \\ address)
}

April 14, 2013

\begin{abstract}
Measurements have been performed on a time-of-flight setup at the Jyväskylä K130 cyclotron, aiming at energy-loss straggling of heavy ions in gases. Theoretical predictions based on recently developed theory as well as an empirical interpolation formula predict that straggling can be more than ten times higher than Bohr straggling in the $\mathrm{MeV} / \mathrm{u}$ regime. Our measurements with up to $9.3 \mathrm{MeV} / \mathrm{u} \mathrm{Kr}$ ions on $\mathrm{He}, \mathrm{N}_{2}, \mathrm{Ne}$ and $\mathrm{Kr}$ targets confirm this feature. Our calculations show the relative contributions of linear straggling, bunching including packing, and charge exchange. Our results for stopping cross sections are compatible with values from the literature.
\end{abstract}

PACS. 34.50.Bw Energy loss and stopping power - 34.70.+e Charge transfer - 34.80.Dp Atomic Excitation and Ionization - 29.40.-n Radiation detectors

\section{Introduction}

The stopping of charged particles in matter is characterized primarily by the mean energy loss and secondly by the fluctuation (straggling). Both quantities are well studied for light ions such as hydrogen and helium [1]. For heavier ions, knowledge of the mean energy loss has improved substantially during the past 10-15 years through both experimental and theoretical studies [2], while knowledge of straggling is still rather fragmentary $[3,4]$. This is due to complexities both on the experimental and the theoretical side.

Critical points in experiments are the quality of the target, accurate knowledge of target thickness and composition, detector resolution and energy spread of the incident beam. For solid targets an additional major obstacle lies in sample preparation and nonuniform foil thickness. These problems become more serious as the stopping cross section increases with increasing atomic number of the penetrating ion, so that rather thin target foils are needed for reliable measurements. Thus, reported high values of straggling in solids deserve caution.

Nevertheless, experiments at very high energies where nonuniform target thickness should not be a problem, indicate that the variance of an energy-loss profile may exceed the Bohr prediction [5] - which provides a useful estimate for light ions - by at least a factor of ten [6].

On the theoretical side several phenomena contribute to straggling beyond the classical result of Bohr. We distinguish between linear and nonlinear straggling:

1. Linear straggling is the fluctuation due to excitation and ionization of independent target electrons. The theory is closely analogous to that for the mean energy loss. A zero-order result is the Bohr formula [5],

$$
\left\langle(\Delta E-\langle\Delta E\rangle)^{2}\right\rangle_{\mathrm{Bohr}} \equiv \Omega_{\mathrm{Bohr}}^{2}=4 \pi Z_{1}^{2} Z_{2} e^{4} N x,
$$

where $Z_{1}$ and $Z_{2}$ are atomic numbers of ion and target, respectively, and $N x$ is the number of atoms per unit target area. Corrections due to electron binding, orbital motion, projectile screening and projectile excitation need to be applied at low beam energies $[7,8]$. Relativistic corrections become important in the opposite limit [9].

2. Nonlinear straggling goes roughly quadratically with the stopping cross section and is made up by

- bunching due to the spatial proximity of electrons in the target atoms $[7,10]$,

- packing due to regular interatomic spacing of atoms in molecules, crystals or amorphous materials $[11$, 12], and 
- charge-exchange straggling due to fluctuations in mean energy loss [13].

A common reference standard for straggling measurements is an empirical interpolation formula by Yang et al. [3]. With regard to heavier ions, this formula was based on a very limited set of available experimental data. Nevertheless, it predicts straggling to reach values far above the Bohr prediction over a certain energy range.

Recent estimates of bunching and packing [14] as well as charge-exchange straggling [15] predict pronounced peaks in energy-loss straggling for heavy ions, going as $S^{2}$ or $(d S / d q)^{2}$, respectively, where $S$ is the stopping cross section and $q$ the ion charge.

While bunching and charge exchange deliver positive contributions to straggling, the packing effect may either enhance (for molecular gases) or diminish (for condensed targets) the fluctuation due to linear straggling. In order to avoid a negative packing correction we perform measurements on gas targets. In the present paper we describe the experimental technique and report first results on measured energy losses and straggling for Kr ions with energies above $1 \mathrm{MeV} / \mathrm{u}$ in gases.

\section{Experimental}

The measurements were carried out at the K130 cyclotron in the Accelerator Laboratory of the University of Jyväskylä. The experimental configuration is illustrated schematically in Figure 1. $\mathrm{A}^{82} \mathrm{Kr}^{22+}$ ion beam with ${ }^{1} 9.3 \mathrm{MeV} / \mathrm{u}$ was employed in the measurements. A retractable grid consisting of thin copper wires (a wire harp) could be inserted to produce ions with a range of lower energies and different charge states [16]. The energy range of interest is selected by means of a $30^{\circ}$ magnetic dipole. The energy loss of each ion was measured by the Time-of-Flight (ToF) method [4]. In these measurements, four carbonfoil time detectors of the Busch et al type [17] made up a pair of Time-of-Flight detector telescopes $\left(\mathrm{ToF}_{1}, \mathrm{ToF}_{2}\right)$. $\mathrm{ToF}_{1}$ tags each ion with its energy, using the known mass prior to entering the gas cell. $\mathrm{ToF}_{2}$ records the ion energy after the gas cell. This is quite similar to the procedure employed by Geissel et al. [18]. The time resolution was determined to be 80 and 100 ps FWHM for $\mathrm{ToF}_{1}$ and $\mathrm{ToF}_{2}$, respectively. The relative energy resolution, $\Delta E / E$ in the $E_{1}$ measurements corresponds to $\sim 0.0025$ at $2 \mathrm{MeV} / \mathrm{u}$ and increases monotonically to $\sim 0.0055$ at $10 \mathrm{MeV} / \mathrm{u}$. The relative energy resolution for $E_{2}$ will be similar. The windows of the gas cell are $2 \mathrm{~mm} \times 2 \mathrm{~mm}$ silicon nitride membranes $^{2}$ on a Si support with a nominal thickness of $200 \mathrm{~nm}$. The length of the gas cell was $0.241 \mathrm{~m}$. The entire system up to the magnet was aligned optically, and the size of the beam was defined by two $1.5 \mathrm{~mm}$ diameter apertures at either end of the gas cell, onto which the silicon nitride windows were glued. The gas pressure was

\footnotetext{
1 The unit $\mathrm{MeV} / \mathrm{u}$ denotes the energy per atomic mass number.

${ }^{2}$ Silson Ltd, http://silson.com
}

measured by a piezoelectric manometer with $1 \%$ absolute accuracy.

\section{Data Analysis}

Measurements were performed with krypton ions in noble gases and nitrogen. For each gas and energy we took data for at least three different gas pressures. $\mathrm{ToF}_{1}$ and $\mathrm{ToF}_{2}$ spectra were first converted into energy spectra $E_{1}$ and $E_{2}$ with the direct beam as a reference.

Two sets of measurements were done which differ in the first step of the data analysis.

I) The direct beam of $9.3 \mathrm{MeV} / \mathrm{u}$ allows to directly extract peak position and width from the $E_{2}$ spectrum.

2) The wire harp in front of the selecting dipole magnet produces ions with different charge states and a continuum of energies. As the magnet separates ions according to momentum over charge, distinct small spans of energies with different charge states may be selected. Ions are grouped into peaks according to their incident energy $E_{1}$ on the gas target measured with $\mathrm{ToF}_{1}$. The grouping in the raw $\mathrm{ToF}_{1}$ data is seen in Figure 2 and after transformation to energy $E_{1}$ in Figure 3. The different path lengths through the wire harp (inset Figure 1) and angular spreading of paths through the magnetic sector lead to a significant $E_{1}$ spread for each group which manifests itself as a sloping correlation between $E_{1}$ and $E_{2}$ (green points in Figure 3 ). This correlation, which would otherwise give rise to an additional energy broadening, was removed on an ion by ion basis by applying a correction to each individual $E_{2}$ value according to the correlation trend line (blue line in Figure 3) depending on the difference of each $E_{1}$ value from the modal $E_{1}$ value for each energy group. The effect of this correction is seen as the red data points in Figure 3. As the energy range in each group is narrow, the change in energy-loss straggling is small and well below experimental uncertainty.

- In the time to energy transformation a correction to the amplitude of the energy data was applied to account for the variations between bin widths in the $\mathrm{ToF}_{1}$ and $\mathrm{ToF}_{2}$ data and the $E_{1}-E_{2}$ data.

A common procedure in the subsequent analysis is to fit a Gaussian to the measured spectrum and from there to find the mean value and variance. Caution is needed here due to the following points:

- According to Bohr [19], an energy-loss profile approaches Gaussian shape for $\Omega \gg T_{\max } \simeq 2 m v^{2}$. This condition would seem well satisfied in principle, with $2 m v^{2}=$ $0.02 \mathrm{MeV}$ at $\mathrm{E}=9.3 \mathrm{MeV} / \mathrm{u}$ and $\Omega$ ranging from 1 to $4 \mathrm{MeV}$ according to figure 5. However, the approach to Gaussian shape has been found to be rather slow $[20,21]$ on the basis of linear straggling theory. Quantitative information incorporating nonlinear straggling is currently unavailable, but since skewness is caused by close collisions, it must show up primarily in linear straggling, while bunching and packing must be secondary. 
- Figure 4 shows energy spectra for Kr in He determined as described above for three different gas pressures. It is seen that there are tails at the $\sim 10 \%$ level which are asymmetric. However, tails are present already in the zero-pressure profile (right).

- If the slight change of the profiles from pressure 149.7 to 221.5 mbar were significant, it would imply a development away from Gaussian shape.

- $\Omega^{2}$ is expected to be proportional to the gas pressure. Therefore, plotting the square of the width is a test on the validity of the approximation. Such a comparison is shown in figure 5. Within the scatter of the experimental points we find it justified to force the straight-line fit to go through the origin.

- A two-parameter fit to data like those shown in figure 5 produces a nonvanishing intercept in some cases. An example is the case of $\mathrm{Kr}$-He shown in the figure. If this effect is real, and not just due to experimental scatter, the straggling parameter extracted from that set of data would be higher.

While caution is indicated, we find it justified to identify the difference between the peak positions in figure 4 as the mean energy loss. This is confirmed by comparison of stopping cross sections with data from the literature shown in figures 6 - 9, where we find agreement with experimental data from refs. [22-24] within 10-15\%. Similar agreement is found with output from the empirical interpolation code SRIM [25], which relies heavily on the same data from refs. [22-24]. The experimental error of our measurements arising primarily from the length of the gas cell, and secondarily from purity of the gas and pressure measurement, has been estimated to be $\lesssim 5 \%$. We also included theoretical curves calculated by the PASS code [26], details below. Two options for the equilibrium charge were chosen, since stopping cross sections tend to become increasingly sensitive to the ion charge with increasing atomic number $Z_{1}[27]$.

The straggling is deduced from the halfwidths of the two spectra. Effects on the peak width from initial beam energy spread, windows of the gas cell and detector resolution are corrected for by subtracting the variance of the spectrum without gas from the respective values with gas.

Experimental errors are derived from the Gaussian fits in plots like figure 5. Scatter of data points within a measurement series and between data sets indicate that there is a systematic error in the range of $15-20 \%$ that is not shown in figures $13-16$. This error is small compared to the reported enhancement of Bohr straggling.

\section{Theory}

\section{Stopping Cross Sections}

Theoretical stopping cross sections shown in figures 6 9 were evaluated with standard PASS input [2], except that Hartree-Fock charge distributions according to ref. [28] instead of hydrogenic wave functions were used for all targets. Two different options were applied for the mean equilibrium charge state as a function of energy [26], a function which we denote as the standard Thomas-Fermi charge state,

$$
q_{\mathrm{TF}}=Z_{1}\left(1-e^{-v / Z_{1}^{2 / 3} v_{0}}\right)
$$

as well as Shima's expression, which also depends on the target material [29].

\subsection{Linear Straggling and Bunching}

Figure 10 shows relative straggling versus beam energy of krypton ions in noble gases and atomic nitrogen predicted by PASS [14]. Thin dashed lines denote linear straggling, including binding, orbital motion and projectile screening, calculated with the same input as stopping cross sections in figures 6 - 9, but only with standard Thomas-Fermi charge states. It is known that linear straggling is less sensitive to the ion charge since it is determined mainly by close collisions [14]. In thick solid lines the bunching effect has been added on the basis of eq. (19) in ref. [14], again with the same input, except that the inverse-Bloch correction that leads over to the Bethe regime at high speed was omitted. Impact-parameter-dependent energy losses were evaluated according to ref. [30] with shell corrections evaluated fully-numerically.

In accordance with previous experience [1], no BetheLivingston maximum - i.e., a maximum due to orbital motion well-established for light ions - is seen in linear straggling. On the other hand, a rather pronounced maximum is found in the bunching effect which, as to be expected, increases with increasing number of target electrons.

\subsection{Charge-Exchange Straggling}

In ref. [31] a fairly general expression was derived for the contribution of charge exchange to straggling. The five curves for $\mathrm{Kr}$ in He in figure 11 are all based on this expression but involve different approximations. All of them neglect the energy loss in charge-changing events.

For a system with only two charge states the general expression reduces to a wellknown formula by Efken et al. [32]. The curve labelled 'Charge 35 and 36' is based on that formula, ignoring all charge states except $35+$ and $36+$. The curve 'Two leading charge states' is based on the same formula but involving only the two charge states next to the peak charge at the respective energy as determined by ETACHA. The curves 'Charge 36, 35 and 34' and 'Three leading charge states' refer similarly to the specific case of a formula derived in ref. [31] for three charge states.

For an arbitrary number of charge states the general expression was reduced in ref. [15] to

$$
\frac{d \Delta \Omega^{2}}{d(N x)}=2\left(\frac{d S}{d q}\right)^{2} G_{0}
$$


where $d S / d q$ is the derivative of the stopping cross section with respect to the ion charge, taken at equilibrium, and

$$
\begin{aligned}
G_{0} & =\sum_{j} F_{j}\left(q_{j}-q\right) \beta_{j}, \\
\beta_{j} & =\sum_{k} q_{k} \int_{0}^{\infty} d x\left(F_{k \ell}(x)-F_{\ell}\right) .
\end{aligned}
$$

Here $F_{k \ell}(x)$ is the charge fraction in charge state $\ell$ at pathlength $x$ for ions ininitially in charge state $k$ at pathlength 0 , and $F_{\ell}$ is the equilibrium charge fraction in state $\ell$.

The most important simplifications underlying eq. (3) are the restriction to charge equilibrium and a smooth dependence of the stopping cross section on the ion charge.

Equation (3) separates into a factor depending on the stopping cross section, which again has been evaluated by the PASS code, while the factor $G_{0}$ is entirely determined by charge fractions. Charge fractions have been evaluated by the ETACHA code [33], because the code developed by two of us [34] is not yet ready for use with ions like krypton. On the other hand, krypton lies on the upper end of the capability of the ETACHA code, and predictions in the energy range of $1-10 \mathrm{MeV} / \mathrm{u}$ are not accurate, as may be seen from figure 12 .

From figure 11 it is seen that all curves nearly coincide at energies above $50 \mathrm{MeV} / \mathrm{u}$. Discrepancies are seen at lower energies. Clearly, the curves 'Charge 35 and 36' and 'Charge 36, 35 and 34' must fail as the mean charge state decreases and the charge spectrum gets broader. From a physical point of view the continuum approximation, which refers to eq. (3), should provide the most realistic estimate. However, the numerical evaluation is tricky, as was pointed in ref. [15]. We note in particular a big discrepancy between the continuum approximation and two of the other approximations at energies below $5 \mathrm{MeV} / \mathrm{u}$ in the sign of the slope. This point is going to be the subject of a separate theoretical study.

Even in consideration of a substantial uncertainty we may nevertheless conclude that according to theory, charge exchange straggling is the dominating contribution to straggling for $\mathrm{Kr}$ ions in the energy range $1 \mathrm{MeV} / \mathrm{u} \lesssim E \lesssim 100$ $\mathrm{MeV} / \mathrm{u}$.

\section{Results}

Figures 13 - 16 show our experimental results for $\mathrm{Kr}$ in $\mathrm{He}, \mathrm{N}_{2}, \mathrm{Ne}$ and $\mathrm{Kr}$ compared with our estimates as well as the empirical interpolation formula of Yang et al. [3]. Data are also shown in table 1.

Almost all experimental results for $\Omega^{2}$ lie a factor of 10 or more above the Bohr value, with maximum values of $(88 \pm 8) \Omega_{\mathrm{Bohr}}^{2}$ for $\mathrm{He}, 11 \pm 2$ for $\mathrm{N}_{2}, 14 \pm 2$ for $\mathrm{Ne}$ and $20 \pm 8$ for $\mathrm{Kr}$ (figures 13 - 16). While the straggling ratio increases almost monotonically with increasing energy for He, it stays essentially constant over the energy range covered by measurements for the other targets.

Theoretical results lie consistently below the measurements. This leaves room for improvement: Energy loss in charge exchange has not yet been taken into account either here nor elsewhere in the literature to our knowledge. We also note that to the extent that tails in the measured spectra could be real, experimental values would become even greater. However, fluctuations in target thickness that may be significant in measurements in solids - do not contribute here: There is a small effect from the windows, but that drops out when the difference is taken between the empty and the filled chamber.

The empirical formula by Yang et al. [3] comes closer to the measurements as far as the magnitude of the straggling ratio is concerned. Although this could be expected from a formula that is based on interpolation of measured results, not many data were available in our energy range at the time. The energy dependence agrees reasonably well with the experimental finding for He. However, roughly the same energy dependence is predicted by the Yang formula for the other targets, while the approximate independence of the beam energy is better described by theory.

Comparison between the theoretical results for He and $\mathrm{Kr}$ targets indicates that the large ratio for He is predominantly due to charge-exchange straggling, while that effect contributes less than $10 \%$ in case of $\mathrm{Kr}$ up to $7 \mathrm{MeV} / \mathrm{u}$.

\section{Comparison with previous measurements}

There are not many experimental data on straggling altogether, considering the number of studies of stopping over the years. Amongst existing studies of straggling, solid targets dominate. Moreover, studies on gas targets often refer to light ions (H, He, Li) and/or low energies [10,35-37].

Sykes et al. [38,39] presented data for fission fragments in gas mixtures but did not refer to theoretical estimates.

Schmidt-Boecking [40] measured straggling of $\sim 0.3-2$ $\mathrm{MeV} / \mathrm{u} \mathrm{Cl}$ ions in an Ar- $\mathrm{CH}_{4}$ gas mixture and concluded that his halfwidths lay about a factor of two above the Bohr value.

Geissel et al. [41,42] measured mean energy losses and straggling for several ions in gases and solids and energies from $0.5-10 \mathrm{MeV} / \mathrm{u}$. Although the main topic in that study has been the density effect in the mean energy loss, straggling data are reported in noble gases and nitrogen for $\mathrm{Pb}, \mathrm{Xe}$ and $\mathrm{Kr}$ ions, several of which lie an order of magnitude above the Bohr value. A single data point, included in figure 16, indicates that the two sets of measurements are compatible.

Schmelmer et al [43] measured straggling of $1 \mathrm{MeV} / \mathrm{u}$ $\mathrm{Ni}$ ions in $\mathrm{D}_{2}$, Ar and solid carbon, focusing in particular on the dependence on entrance and exit ion charge. Measured values up to about a factor of 2 above the Bohr value were reported, but a direct comparison with our data is not possible.

\section{Conclusions}

- The present measurements have shown that for krypton ions in the energy range of $2-10 \mathrm{MeV} / \mathrm{u}$, straggling may exceed the Bohr value by 1-2 orders of magnitude. 
- The quantitative results may to some degree depend on the significance of the tails in the time-of-flight spectra. If real, these tails would enhance straggling further.

- Figures 13 - 16 also show that for Kr ions, the straggling ratio measured in $\mathrm{He}$ is an order of magnitude larger than for $\mathrm{N}_{2}$, Ne and $\mathrm{Kr}$. The formula of Yang et al. [3] predicts a less dramatic decrease from He to Kr.

- Quantitative agreement between estimates and experiment as well as between different estimates is still not satisfactory. An important feature still missing is the contribution of the energy lost in charge-changing events, which is the subject of a separate study.

This work has been supported by the EU 7th framework programme 'Integrating Activities - Transnational Access', by the Academy of Finland under the Finnish Centre of Excellence Programme 2012-2017, by the Danish Natural Science Research Council, by the Carlsberg Foundation and by the German Science Foundation DFG (SFB 616: energy dissipation at surfaces). Our friend, the late Hans Henrik Andersen, has given useful input in the initiation and planning of this experiment. Thanks are due to Henri Kivistö, Mari Napari and Laura Mättö for help with the data taking.

\section{References}

1. P. Sigmund, Particle penetration and radiation effects, vol. 151 of Springer Series in Solid-State Sciences (Springer, Berlin, 2006).

2. ICRU, Stopping of ions heavier than helium, vol. 73 of ICRU Report (Oxford University Press, Oxford, 2005).

3. Q. Yang, D. J. O'Connor and Z. Wang, Nucl. Instrum. Methods B 61 (1991) 149.

4. H. Geissel, H. Weick, C. Scheidenberger, R. Bimbot and D. Gardès, Nucl. Instrum. Methods B 195 (2002) 3.

5. N. Bohr, Philos. Mag. 30 (1915) 581.

6. H. Weick, H. Geissel, C. Scheidenberger, F. Attallah, T. Baumann, D. Cortina, M. Hausmann, B. Lommel, G. Münzenberg, N. Nankov, F. Nickel, T. Radon, H. Schatz, K. Schmidt, J. Stadlmann, K. Sümmerer, M. Winkler and H. Wollnik, Nucl. Instrum. Methods B 164-165 (2000) 168.

7. P. Sigmund and A. Schinner, Europ. Phys. J. D 23 (2003) 201.

8. P. L. Grande and G. Schiwietz, Nucl. Instrum. Methods B 267 (2009) 859.

9. J. Lindhard and A. H. Sørensen, Phys. Rev. A 53 (1996) 2443.

10. F. Besenbacher, J. U. Andersen and E. Bonderup, Nucl. Instrum. Methods 168 (1980) 1.

11. P. Sigmund, Phys. Rev. A 14 (1976) 996.

12. P. Sigmund, Mat. Fys. Medd. Dan. Vid. Selsk. 40 no. 5 (1978) 1.

13. O. Vollmer, Nucl. Instrum. Methods 121 (1974) 373.

14. P. Sigmund and A. Schinner, Europ. Phys. J. D 58 (2010) 105.

15. P. Sigmund, O. Osmani and A. Schinner, Nucl. Instrum. Methods B 269 (2011) 804.
16. A. Javanainen, Particle radiation in microelectronics, Ph.D. thesis, University of Jyväskylä (2012), URL http://www.jyu.fi/static/fysiikka/vaitoskirjat/ 2012/Javanainen-Arto-2012.pdf.

17. F. Busch, W. Pfeffer, B. Kohlmeyer, D. Schull and F. Puhlhoffer, Nucl. Instrum. Methods 171 (1980) 71.

18. H. Geissel, P. Armbruster, T. Kitahara, G. Kraft, H. Spieler and K. Güttner, Nucl. Instrum. Methods 170 (1980) 217.

19. N. Bohr, Mat. Fys. Medd. Dan. Vid. Selsk. 18 no. 8 (1948) 1.

20. L. G. Glazov, Nucl. Instrum. Methods B 192 (2002) 239.

21. L. G. Glazov, P. Sigmund and A. Schinner, Nucl. Instrum. Methods B 195 (2002) 183.

22. R. Bimbot, C. Cabot, D. Gardès, H. Gauvin, I. Orliange, L. DeReilhac, K. Subotic and F. Hubert, Nucl. Instrum. Methods B 44 (1989) 19.

23. J. Herault, R. Bimbot, H. Gauvin, B. Kubica, R. Anne, G. Bastin and F. Hubert, Nucl. Instrum. Methods B 61 (1991) 156.

24. H. Paul, Stopping power graphs (2009), URL www.exphys. uni-linz.ac.at/stopping/.

25. J. F. Ziegler, Particle interactions with matter (2012), URL www.srim.org.

26. P. Sigmund and A. Schinner, Nucl. Instrum. Methods B 195 (2002) 64

27. A. Fettouhi, H. Geissel, A. Schinner and P. Sigmund, Nucl. Instrum. Methods B 245 (2006) 22.

28. E. Clementi and C. Roetti, Atomic Data \& Nucl. Data Tables 14 (1974) 177.

29. K. Shima, T. Ishihara and T. Mikumo, Nucl. Instrum. Methods 200 (1982) 605.

30. A. Schinner and P. Sigmund, Europ. Phys. J. D 56 (2010) 41.

31. P. Sigmund, Nucl. Instrum. Methods B 69 (1992) 113.

32. B. Efken, D. Hahn, D. Hilscher and G. Wüstefeld, Nucl. Instrum. Methods 129 (1975) 219.

33. J. P. Rozet, C. Stephan and D. Vernhet, Nucl. Instrum. Methods B 107 (1996) 67.

34. O. Osmani and P. Sigmund, Nucl. Instrum. Methods B 269 (2011) 813.

35. P. Hvelplund, Phys. Rev. A 11 (1975) 1921.

36. H. H. Andersen, F. Besenbacher and H. Knudsen, Nucl. Instrum. Methods 149 (1978) 121.

37. F. Besenbacher, H. H. Andersen, P. Hvelplund and H. Knudsen, Mat. Fys. Medd. Dan. Vid. Selsk. 40 no. 9 (1981) 1.

38. D. A. Sykes and S. J. Harris, Nucl. Instrum. Methods 97 (1971) 203

39. M. B. Al-Bedri and S. J. Harris, Nucl. Instrum. Methods 124 (1975) 125.

40. H. Schmidt-Boecking and H. Hornung, Z. Phys. A 286 (1978) 253.

41. H. Geissel, Y. Laichter, W. F. W. Schneider and P. Armbruster, Nucl. Instrum. Methods 194 (1982) 21.

42. H. Geissel, Y. Laichter, W. F. W. Schneider and P. Armbruster, Nucl. Instrum. Methods 215 (1983) 329.

43. O. Schmelmer, G. Dollinger, C. M. Frey, A. Bergmaier and S. Karsch, Nucl. Instrum. Methods B 145 (1998) 261, erratum ibid. 152 (1999) 409.

44. G. Schiwietz and P. L. Grande, Nucl. Instrum. Methods B 175-177 (2001) 125. 
Table 1. Summary of measured stopping cross sections (estimated error 5\%) and straggling ratios.

\begin{tabular}{|c|c|c|c|c|c|c|c|c|c|c|c|}
\hline \multicolumn{3}{|c|}{$\mathrm{Kr}-\mathrm{He}$} & \multicolumn{3}{|c|}{$\mathrm{Kr}-\mathrm{N}$} & \multicolumn{3}{|c|}{$\mathrm{Kr}-\mathrm{Ne}$} & \multicolumn{3}{|c|}{$\mathrm{Kr}-\mathrm{Kr}$} \\
\hline $\begin{array}{c}\text { Energy } \\
(\mathrm{MeV} / \mathrm{u})\end{array}$ & $\begin{array}{l}S\left(10^{-15}\right. \\
\left.\mathrm{eVcm}^{2}\right)\end{array}$ & $\Omega^{2} / \Omega_{\mathrm{Bohr}}^{2}$ & $\begin{array}{c}\text { Energy } \\
(\mathrm{MeV} / \mathrm{u})\end{array}$ & $\begin{array}{l}S\left(10^{-15}\right. \\
\left.\mathrm{eVcm}^{2}\right)\end{array}$ & $\Omega^{2} / \Omega_{\mathrm{Bohr}}^{2}$ & $\begin{array}{c}\text { Energy } \\
(\mathrm{MeV} / \mathrm{u})\end{array}$ & $\begin{array}{l}S\left(10^{-15}\right. \\
\left.\mathrm{eVcm}^{2}\right)\end{array}$ & $\Omega^{2} / \Omega_{\mathrm{Bohr}}^{2}$ & $\begin{array}{c}\text { Energy } \\
(\mathrm{MeV} / \mathrm{u})\end{array}$ & $\begin{array}{l}S\left(10^{-15}\right. \\
\left.\mathrm{eVcm}^{2}\right)\end{array}$ & $\Omega^{2} / \Omega_{\mathrm{Bohr}}^{2}$ \\
\hline 2.10 & $5.4 \mathrm{E}+2$ & $11.4 \pm 0.3$ & 5.42 & $1.04 \mathrm{E}+3$ & $20.5 \pm 2.4$ & 4.39 & $1.43 \mathrm{E}+3$ & $13.5 \pm 0.8$ & 3.74 & & $11.8 \pm 1.7$ \\
\hline 2.29 & $5.4 \mathrm{E}+2$ & $16.8 \pm 0.2$ & 5.79 & $1.03 \mathrm{E}+3$ & $19.2 \pm 2.4$ & 4.72 & $1.40 \mathrm{E}+3$ & $14.0 \pm 0.2$ & 4.27 & & $19.6 \pm 7.5$ \\
\hline 2.49 & $5.3 \mathrm{E}+2$ & $19.3 \pm 0.2$ & 6.16 & $1.00 \mathrm{E}+3$ & $20.0 \pm 1.8$ & 5.06 & $1.36 \mathrm{E}+3$ & $12.4 \pm 1.9$ & 4.55 & & $18.5 \pm 1.8$ \\
\hline 2.69 & $5.2 \mathrm{E}+2$ & $18.2 \pm 4.1$ & 6.54 & $9.8 \mathrm{E}+2$ & $20.2 \pm 1.4$ & 5.42 & $1.33 \mathrm{E}+3$ & $11.0 \pm 1.3$ & 5.43 & $3.3 \mathrm{E}+3$ & $10.5 \pm 0.2$ \\
\hline 2.90 & $5.1 \mathrm{E}+2$ & $21.4 \pm 2.1$ & 6.94 & $9.5 \mathrm{E}+2$ & $20.3 \pm 1.2$ & 5.78 & $1.30 \mathrm{E}+3$ & $10.9 \pm 1.4$ & 5.79 & $3.3 \mathrm{E}+3$ & $10.93 \pm 0.04$ \\
\hline 3.12 & $5.0 \mathrm{E}+2$ & $24.5 \pm 2.1$ & 9.37 & $8.3 \mathrm{E}+2$ & $21.6 \pm 2.1$ & 6.16 & $1.26 \mathrm{E}+3$ & $12.3 \pm 1.0$ & 6.17 & $3.2 \mathrm{E}+3$ & $11.0 \pm 0.7$ \\
\hline 3.34 & $4.9 \mathrm{E}+2$ & $29.0 \pm 0.2$ & 9.36 & $8.5 \mathrm{E}+2$ & $14.1 \pm 0.8$ & 6.54 & $1.24 \mathrm{E}+3$ & $12.3 \pm 1.9$ & 6.55 & $3.2 \mathrm{E}+3$ & $11.5 \pm 0.5$ \\
\hline 3.58 & $4.8 \mathrm{E}+2$ & $34.0 \pm 0.9$ & & & & 6.94 & $1.20 \mathrm{E}+3$ & $11.8 \pm 1.3$ & 6.95 & $3.1 \mathrm{E}+3$ & $9.48 \pm 0.17$ \\
\hline 3.82 & $4.7 \mathrm{E}+2$ & $40.5 \pm 2.9$ & & & & 7.35 & $1.17 \mathrm{E}+3$ & $10.8 \pm 1.7$ & 9.37 & $2.9 \mathrm{E}+3$ & $9.34 \pm 0.85$ \\
\hline 4.07 & $4.6 \mathrm{E}+2$ & $41.2 \pm 2.7$ & & & & 9.36 & $1.05 \mathrm{E}+3$ & $4.6 \pm 0.2$ & 9.36 & $2.8 \mathrm{E}+3$ & $6.13 \pm 0.51$ \\
\hline 4.32 & $4.6 \mathrm{E}+2$ & $50.7 \pm 7.2$ & & & & & & & & & \\
\hline 4.74 & $4.3 \mathrm{E}+2$ & $39.3 \pm 3.4$ & & & & & & & & & \\
\hline 5.07 & $4.2 \mathrm{E}+2$ & $50.2 \pm 3.0$ & & & & & & & & & \\
\hline 5.43 & $4.1 \mathrm{E}+2$ & $63.5 \pm 3.4$ & & & & & & & & & \\
\hline 5.79 & $4.0 \mathrm{E}+2$ & $72.0 \pm 3.1$ & & & & & & & & & \\
\hline 6.17 & $4.0 \mathrm{E}+2$ & $81.3 \pm 2.8$ & & & & & & & & & \\
\hline 6.55 & $3.9 \mathrm{E}+2$ & $86.3 \pm 2.1$ & & & & & & & & & \\
\hline 6.95 & $3.8 \mathrm{E}+2$ & $85.5 \pm 5.4$ & & & & & & & & & \\
\hline 7.36 & $3.7 \mathrm{E}+2$ & $87.9 \pm 8.5$ & & & & & & & & & \\
\hline 9.37 & $3.0 \mathrm{E}+2$ & $44.6 \pm 3.3$ & & & & & & & & & \\
\hline 9.38 & $3.1 \mathrm{E}+2$ & $47.4 \pm 1.1$ & & & & & & & & & \\
\hline
\end{tabular}




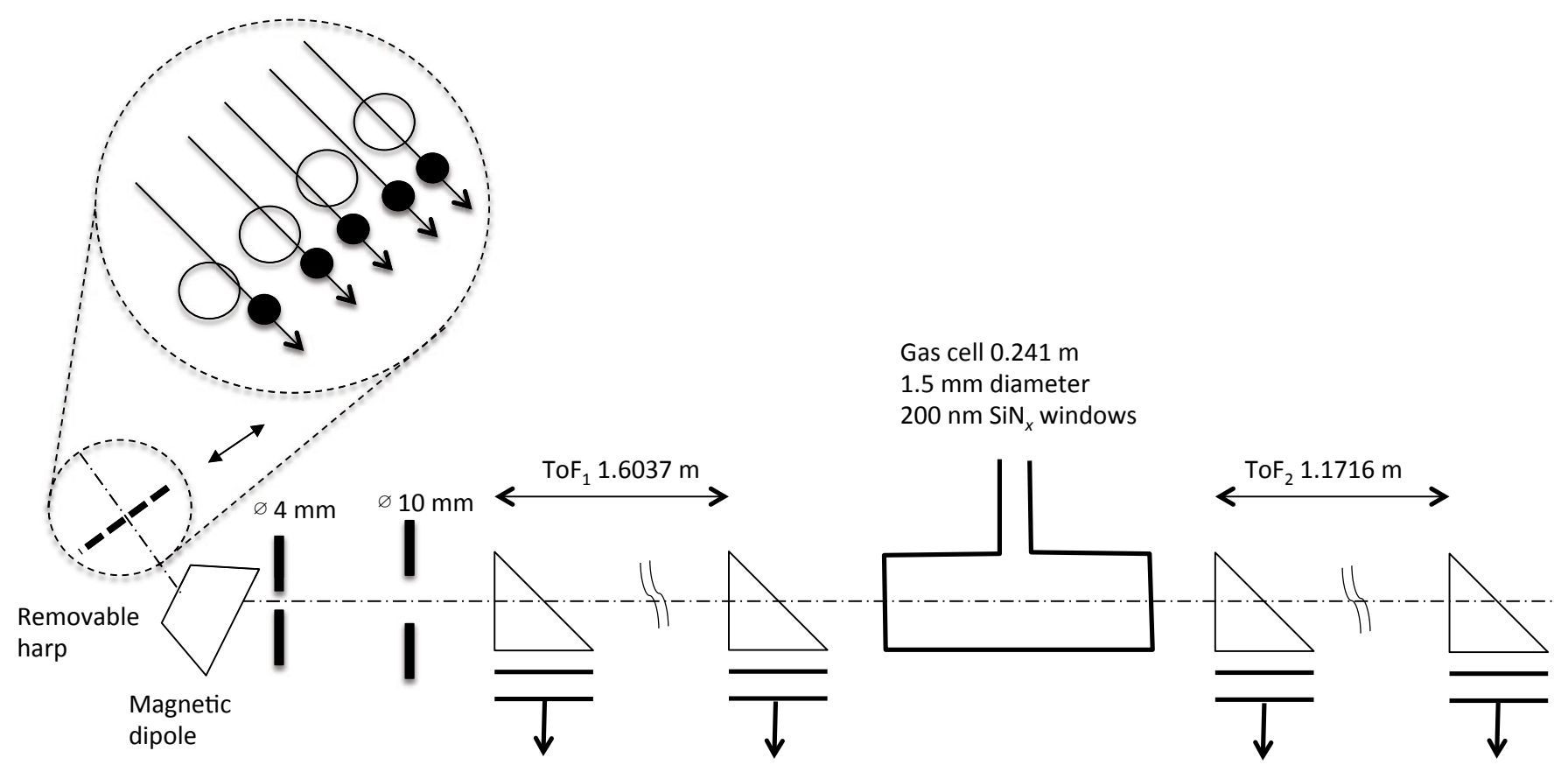

Fig. 1. Experimental configuration. The beam enters from the left. A removable wire harp allows for energy degradation to produce ions with different $M E / q^{2}$ that are selected by the magnetic dipole [16].

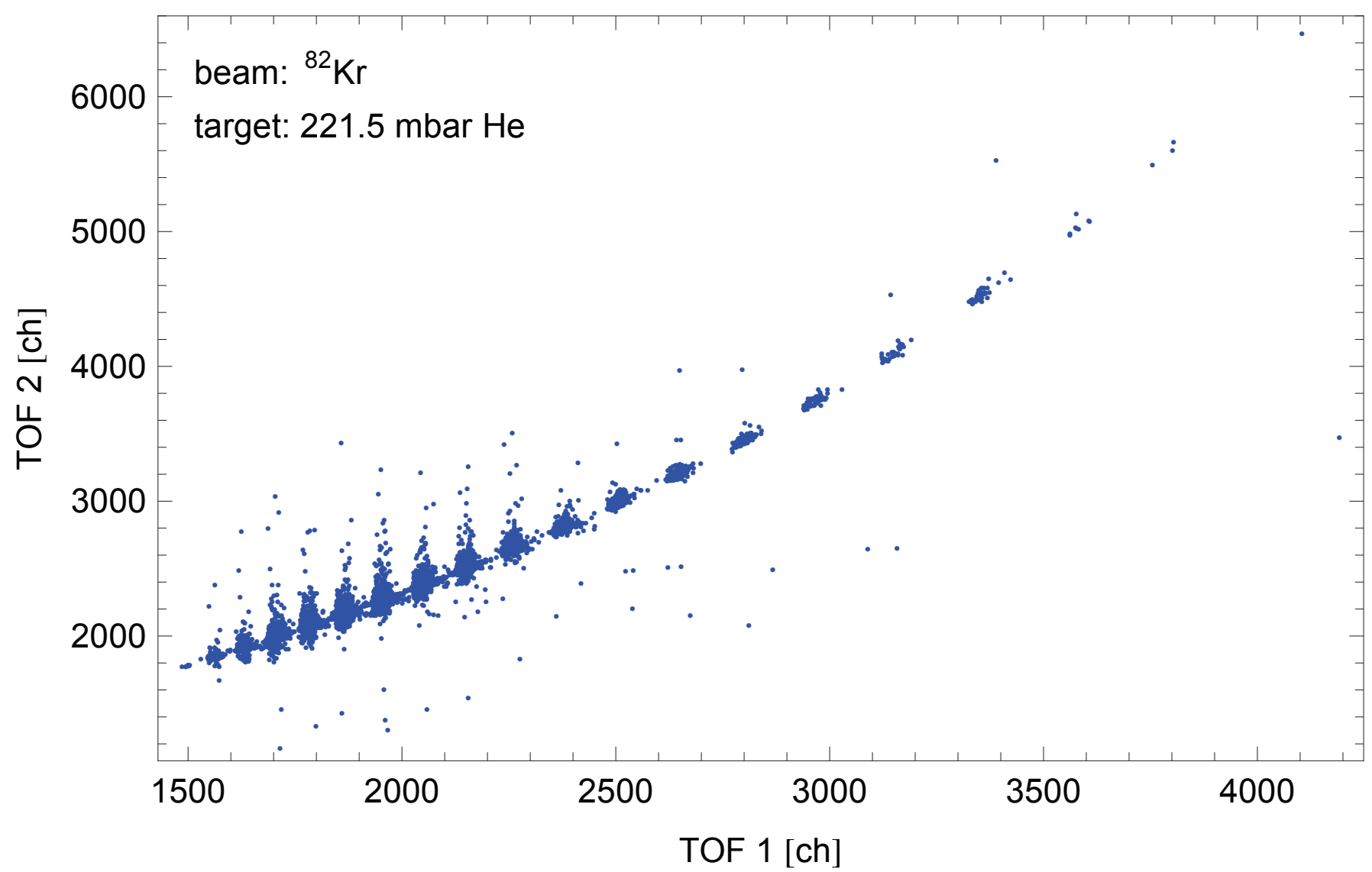

Fig. 2. Scatter plot of $\mathrm{ToF}_{1}$ versus $\mathrm{ToF}_{2}$ for ${ }^{82} \mathrm{Kr}$ on 221.5 mbar He (higher ToF channels correspond to lower energies). Several groups of ion energies are visible. 


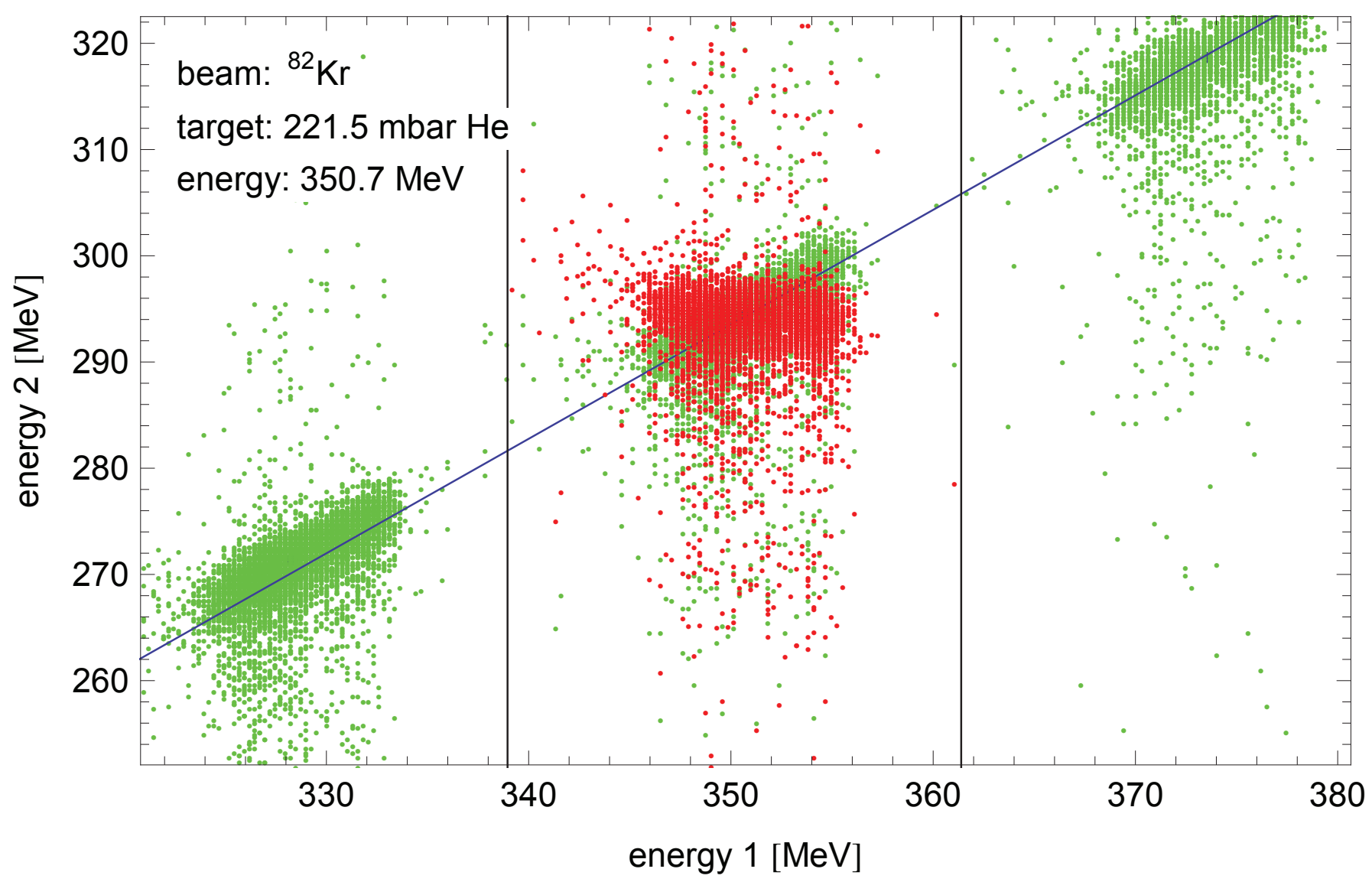

Fig. 3. Scatter plot of energy $E_{2}$ versus energy $E_{1}$ for ${ }^{82} \mathrm{Kr}$ on 221.5 mbar He. Green dots indicate three groups of uncorrected data. The blue line corresponds to the energy correlation between energy 1 and 2 . The red dots belong to the central energy group but are shifted vertically in proportion to the horizontal distance from the peak maximum to correct for the energy correlation. 


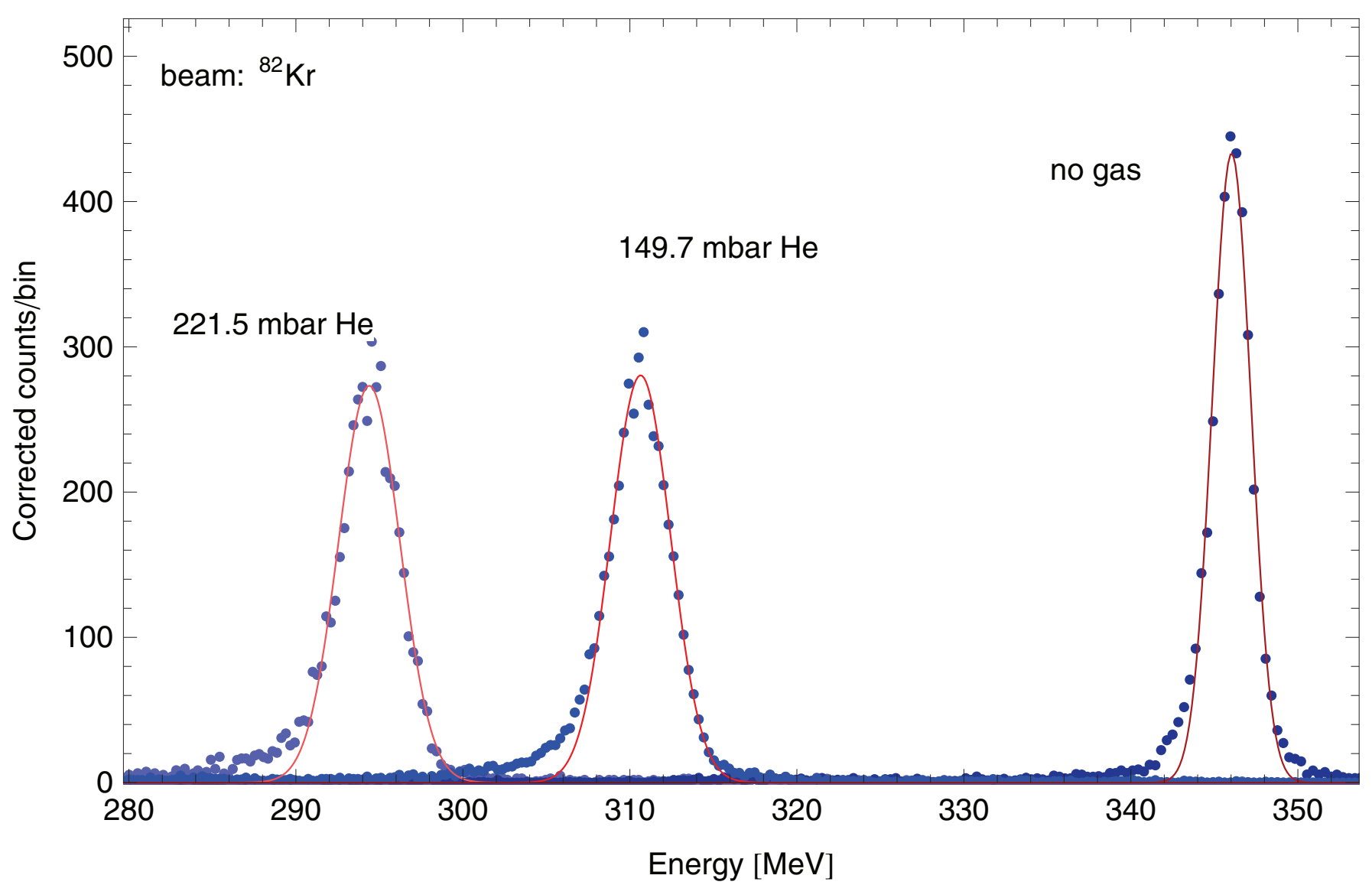

Fig. 4. Energy spectra for $350 \mathrm{MeV} \mathrm{Kr}$ on He at three pressures. 


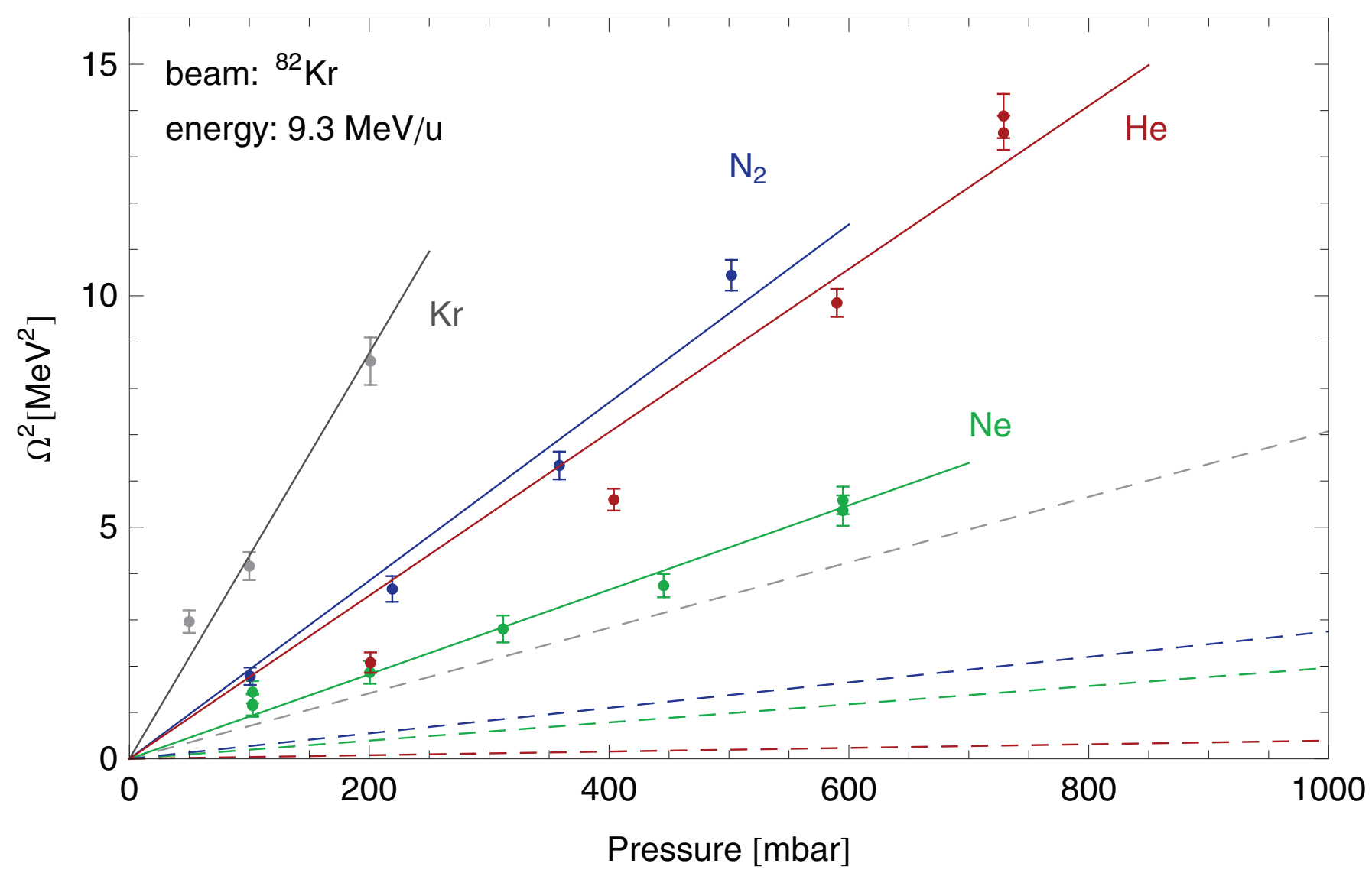

Fig. 5. Straggling $\Omega^{2}$ versus pressure for ${ }^{82} \mathrm{Kr}$ on several gases at $9.3 \mathrm{MeV} / \mathrm{u}$. Solid lines are fits to the data, forced to go through the origin, while dashed lines represent Bohr straggling. 


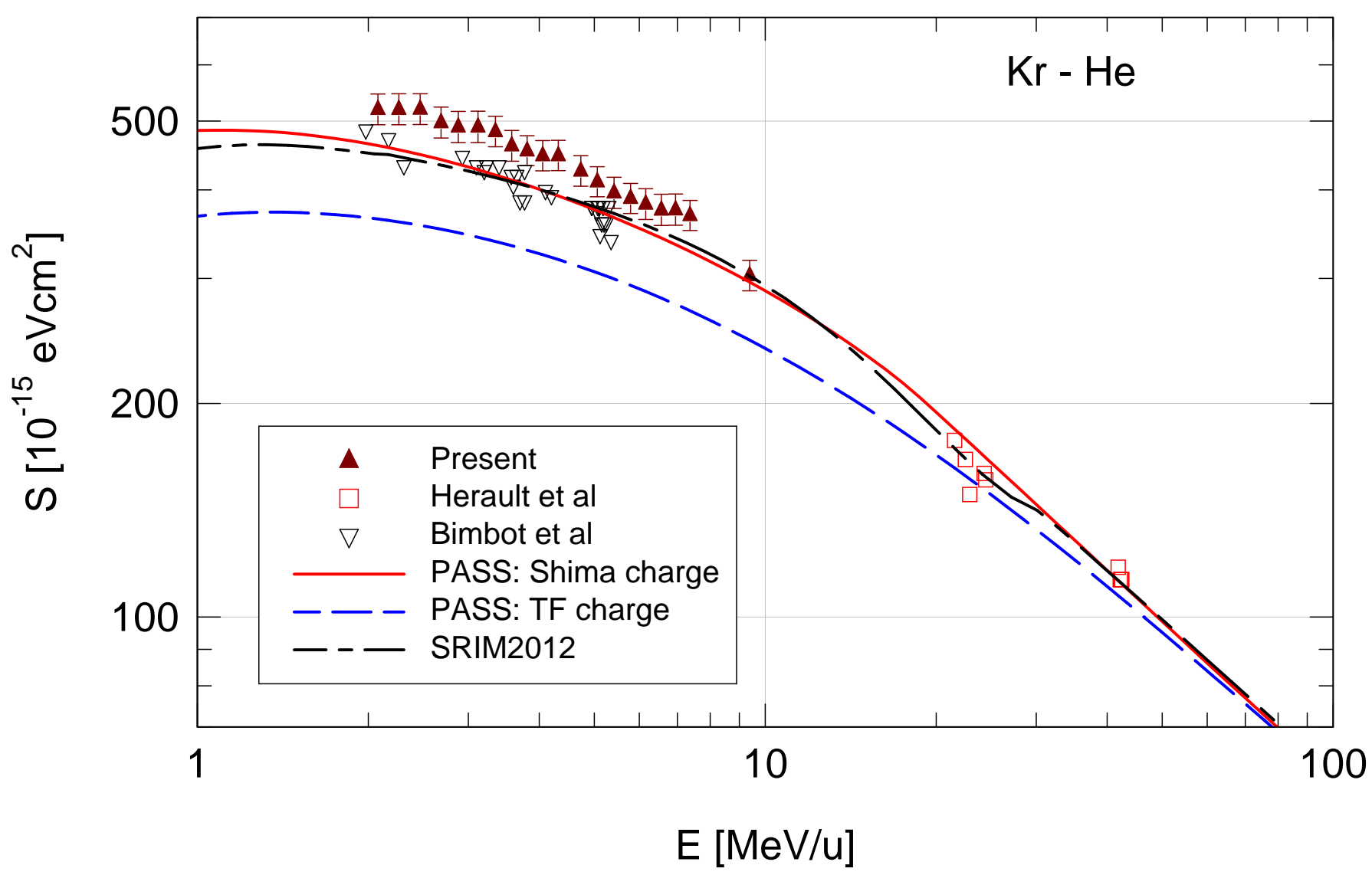

Fig. 6. Stopping cross sections for $\mathrm{Kr}$ in He. Present measurements compared to experimental data from Bimbot et al. [22] and Herault et al. [23]. Also included are data from SRIM [25] and PASS [26], the latter for two different expressions for the mean equilibrium charge specified in ref. [26]. 


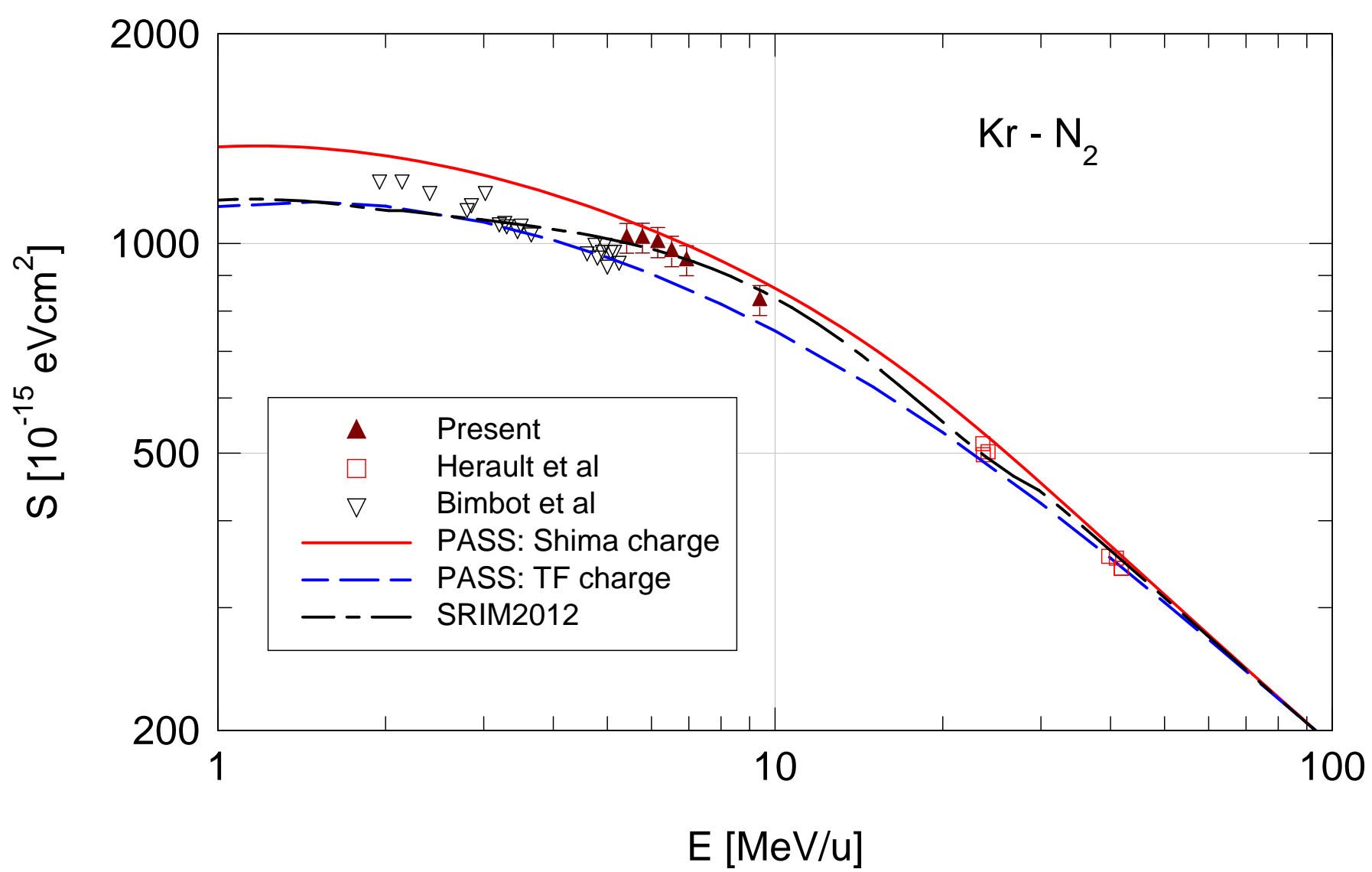

Fig. 7. Same as figure 6 for $\mathrm{Kr}$ in $\mathrm{N}_{2}$. 


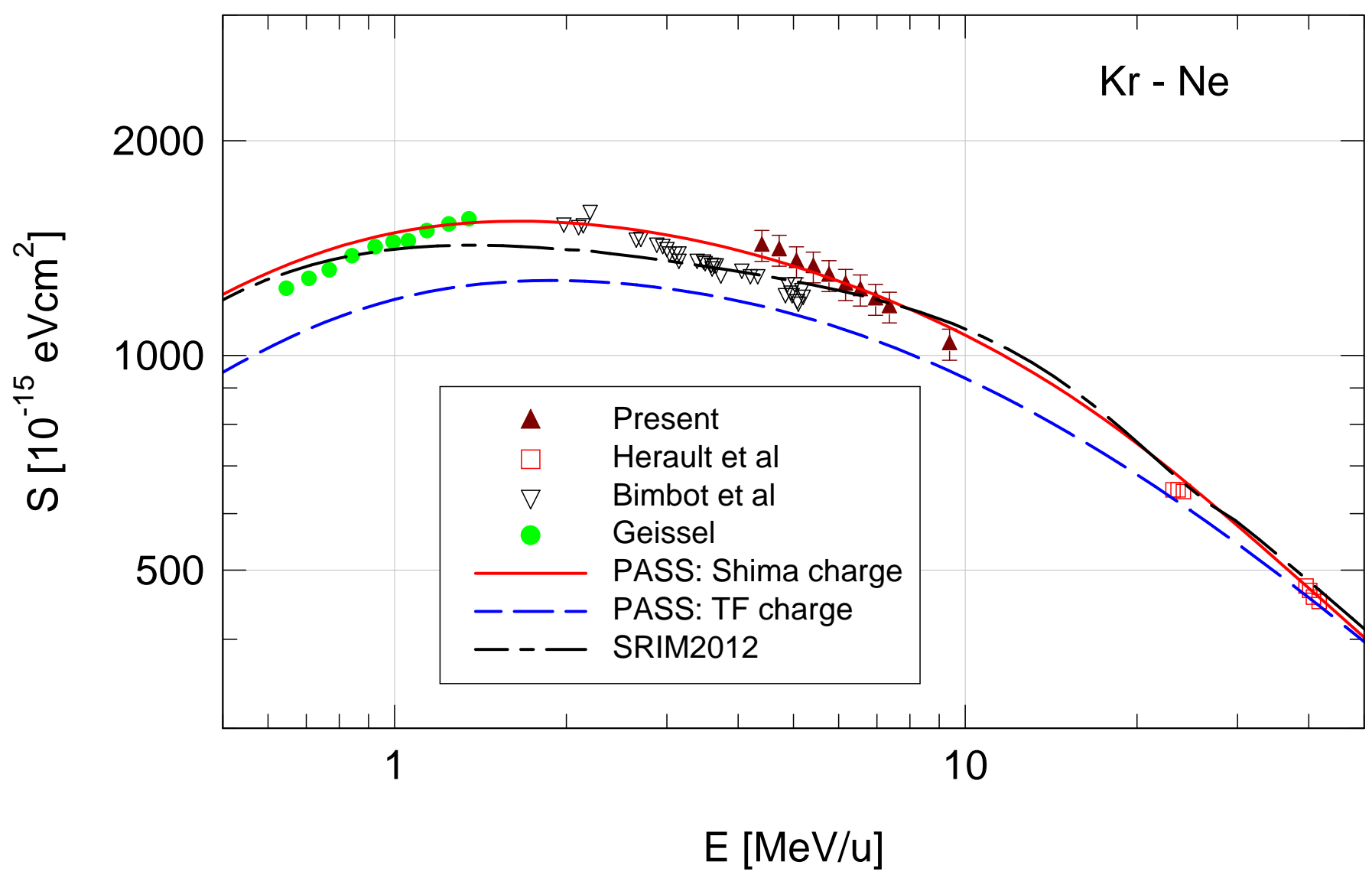

Fig. 8. Same as figure 6 for Kr in Ne. Also included are experimental data from Geissel quoted by Paul [24]. 


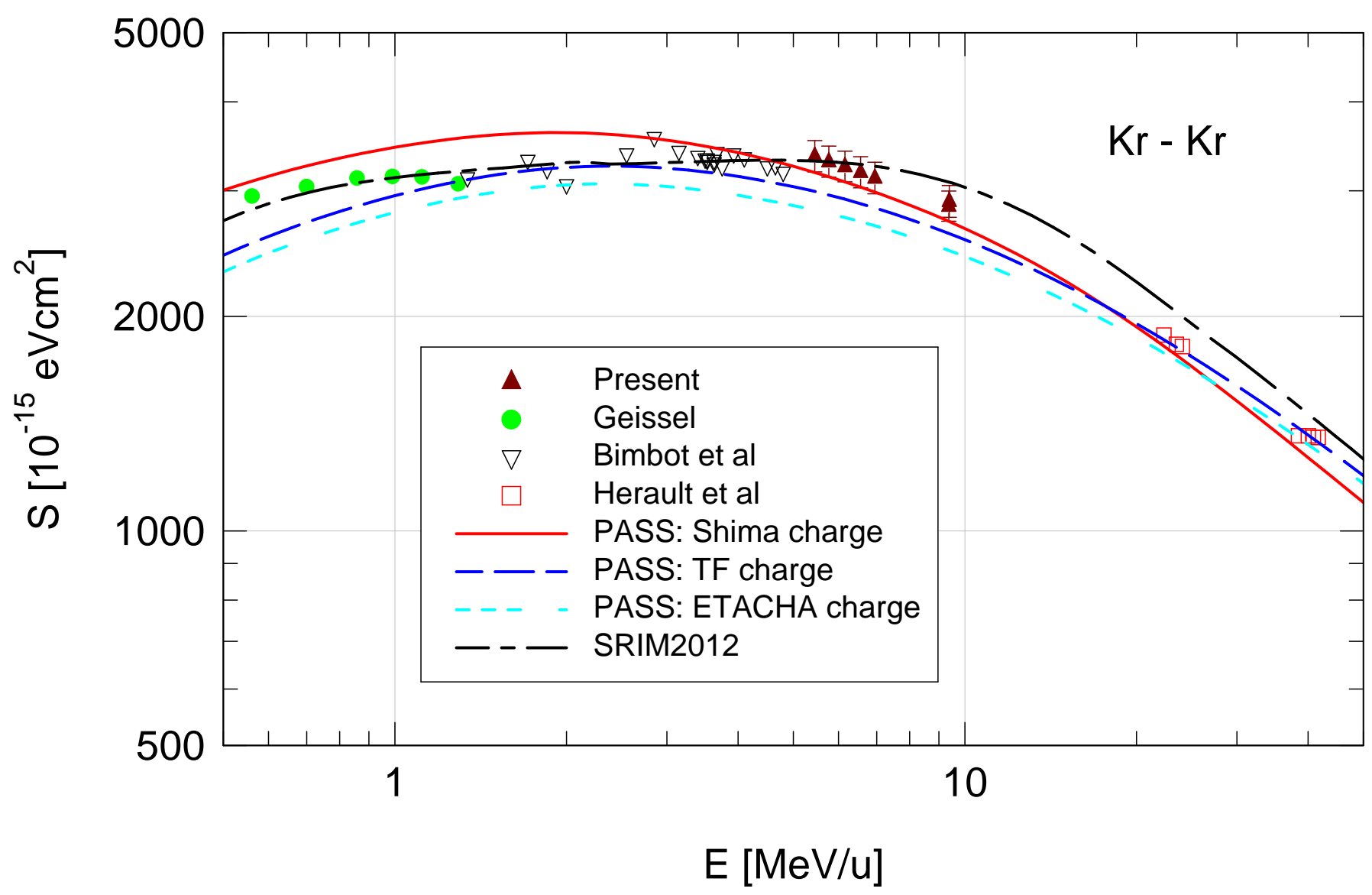

Fig. 9. Same as figure 8 for $\mathrm{Kr}$ in Kr. 


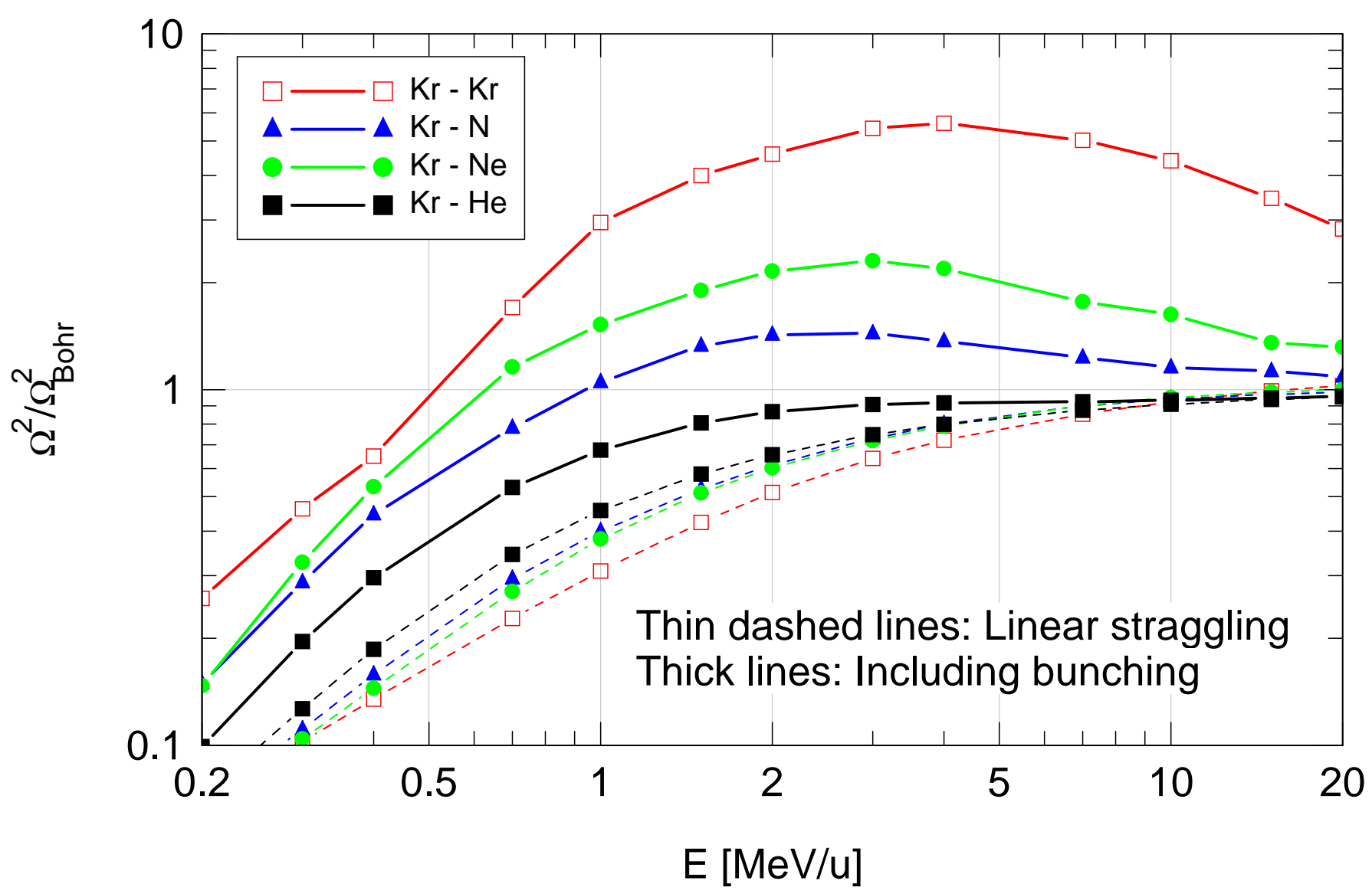

Fig. 10. Straggling of krypton ions in noble gases according to the PASS code [14]. Thin dashed lines denote linear straggling. Thick solid lines: Including bunching. Charge-exchange straggling ignored. 


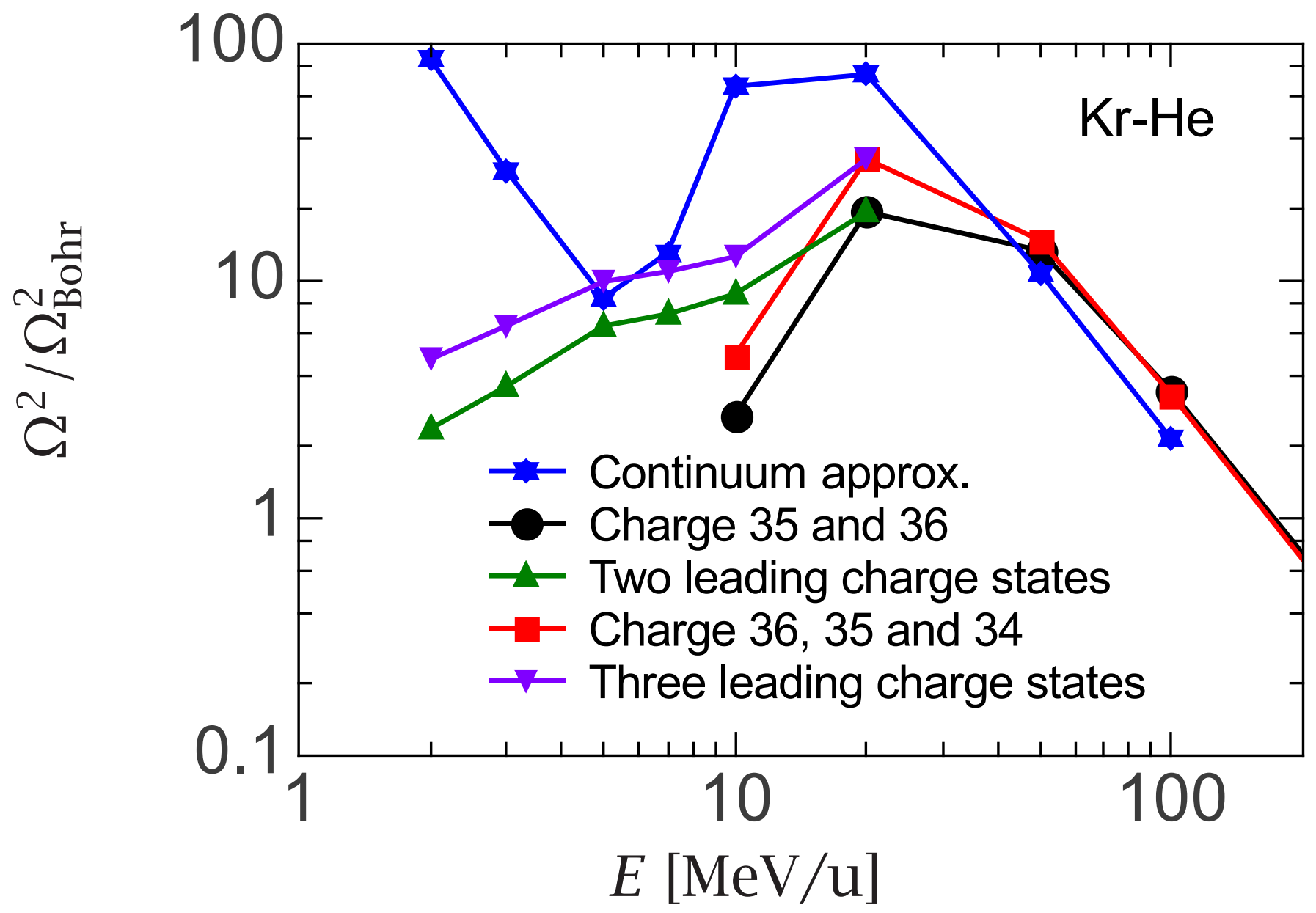

Fig. 11. Charge exchange straggling for $\mathrm{Kr}$ in He. See text. 


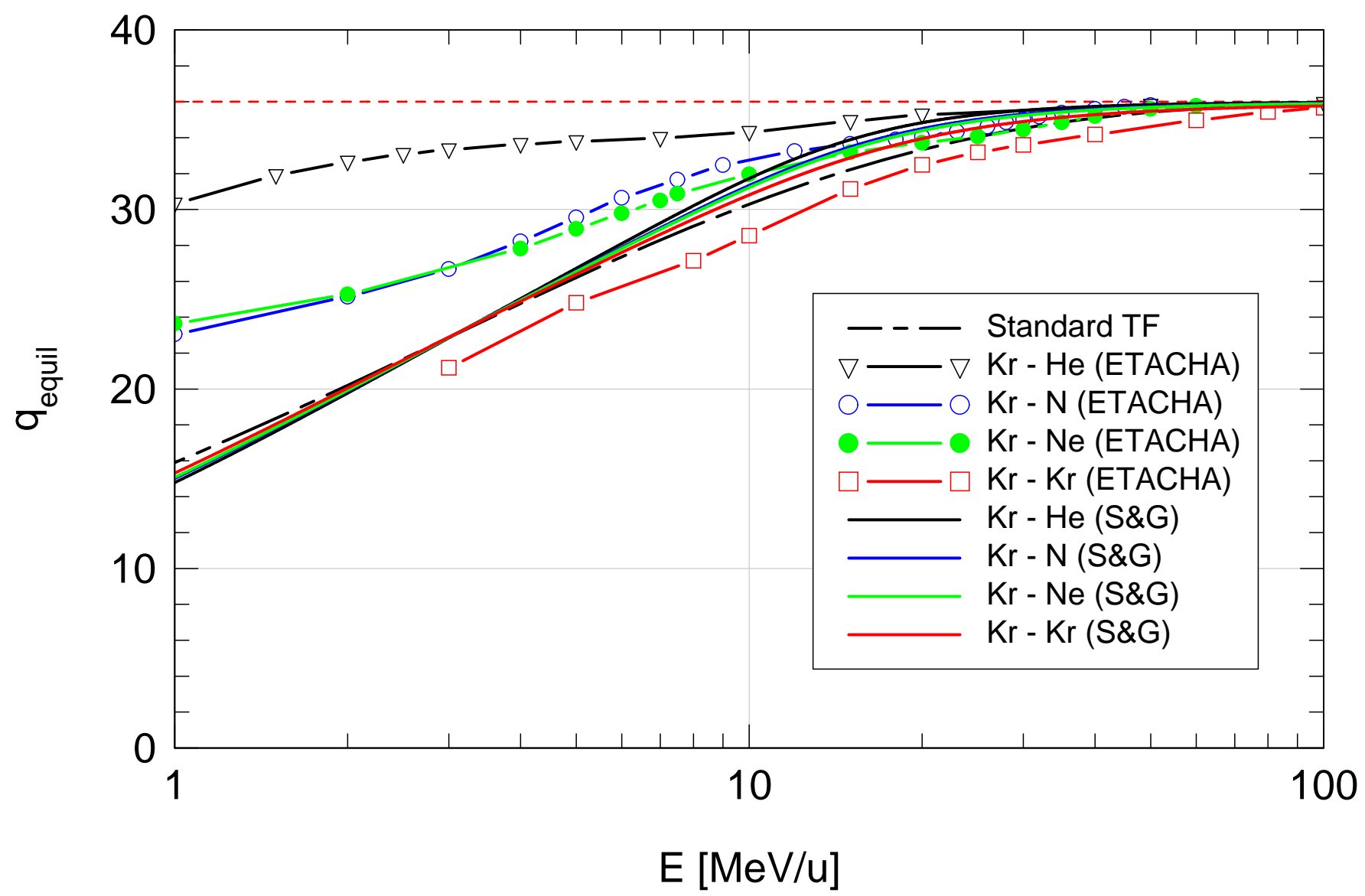

Fig. 12. Equilibrium charge states calculated by ETACHA, compared with the standard Thomas-Fermi charge eq. (2) and the empirical interpolation formula from Schiwietz \& Grande [44]. 


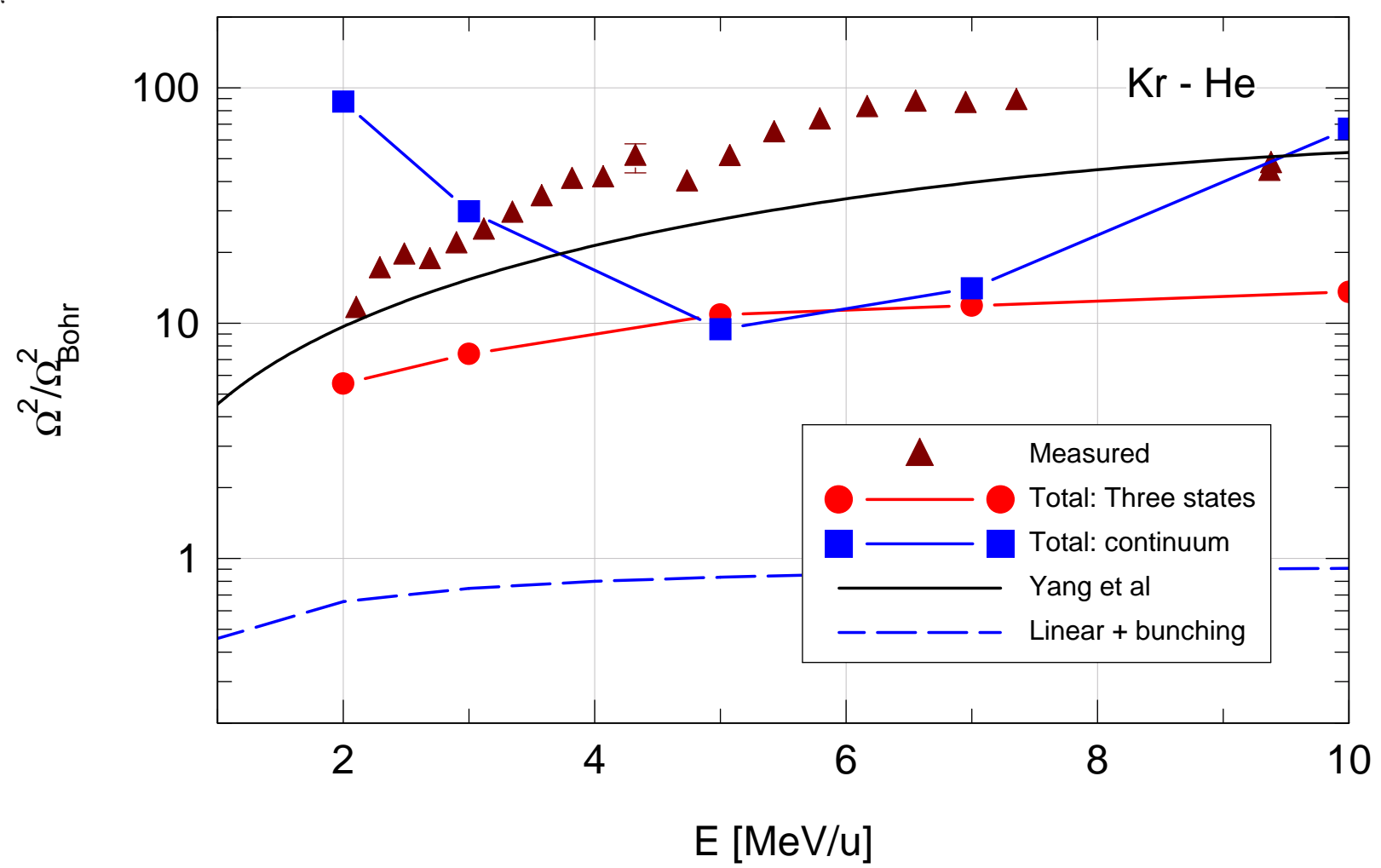

Fig. 13. Relative straggling for $\mathrm{Kr}$ in $\mathrm{He}, \mathrm{N}_{2}, \mathrm{Ne}$ and $\mathrm{Kr}$. Present measurements compared to present calculations (Linear, bunching, packing and charge exchange) as well as empirical formula by Yang et al. [3].

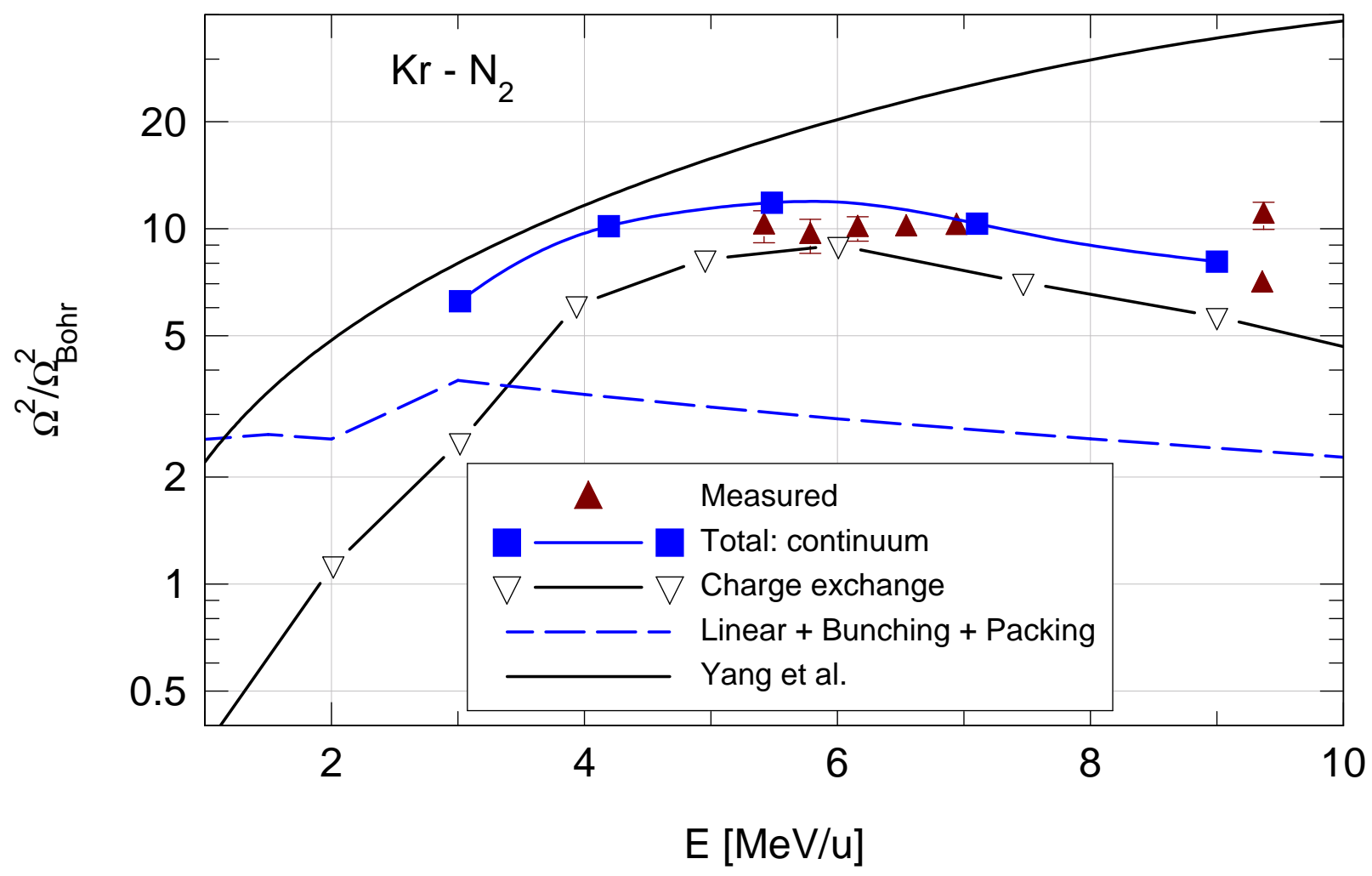

Fig. 14. Same as figure 13 for $\mathrm{N}_{2}$. 


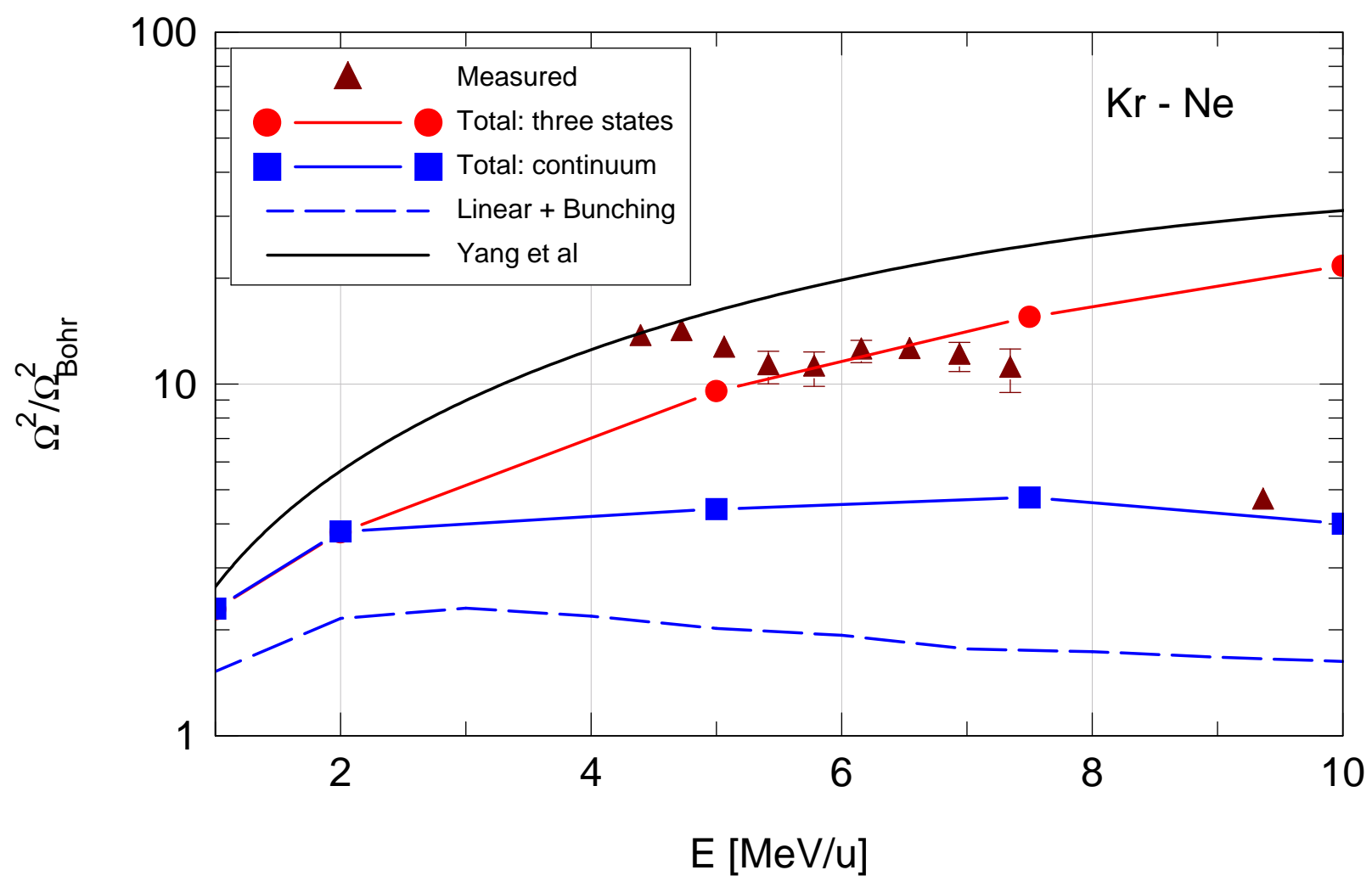

Fig. 15. Same as figure 13 for Ne.

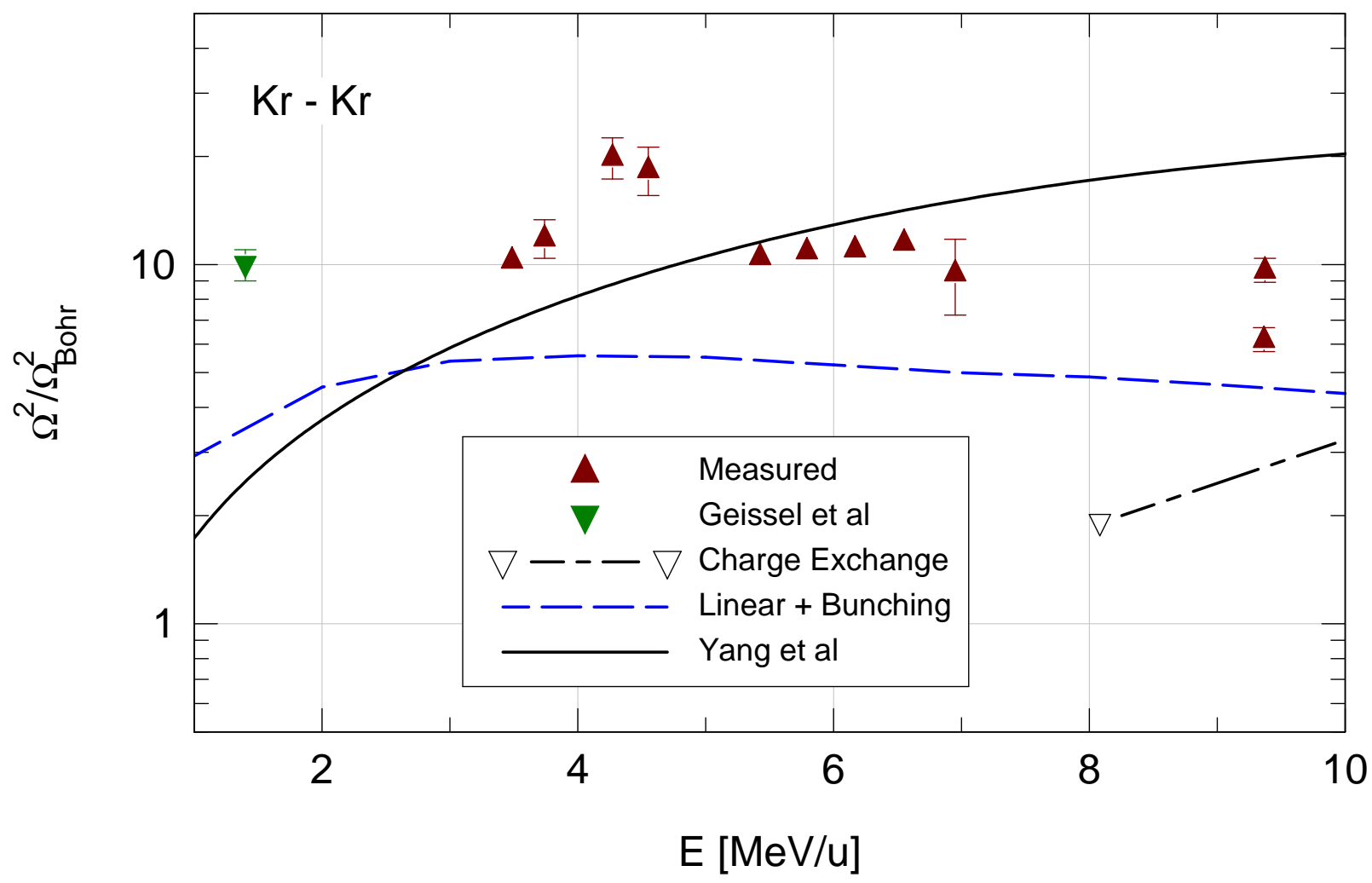

Fig. 16. Same as figure 13 for Kr. A single point from Geissel et al. [41] has also been included. 